\title{
CREATIVITY, EMERGENCE OF NOVELTY, AND SPONTANEOUS SYMMETRY BREAKING
}

\author{
Radek Trnka, Ph.D. ${ }^{1,2}$ \\ Martin Kuška, Ph.D. ${ }^{1,2}$ \\ Inna Čábelková, Ph.D. ${ }^{1,3}$ \\ ${ }^{1}$ Prague College of Psychosocial Studies, Czech Republic \\ ${ }^{2}$ Palacky University Olomouc, Czech Republic \\ ${ }^{3}$ Charles University in Prague, Czech Republic
}

This is the final version of the manuscript that has been published in:

SGEM Conference Proceedings, Volume 5, Issue 2.1, 203-210, ISSN 2367-5659.

\begin{abstract}
The philosophy of mind concerns much about how novelty occurs in the world. The very recent progress in this field inspired by quantum mechanics indicates that symmetry restoration occurs in the mind at the moment when new creative thought arises. Symmetry restoration denotes the moment when one's cognition leaves ordinary internalized mental schemes such as conceptual categories, heuristics, subjective theories, conventional thinking, or expectations. At this moment, fundamentally new, original thought may arise. We also predict that in older age, symmetry restoration is less likely to occur as internalized mental schemes become more rigid in the elderly. Furthermore, the present study demonstrates that symmetry restoration may occur not only individually, in one's mind, but also collectively, during collaborative creative activities, e.g. during small-group brainstorming sessions or creative improvisational performances. The possibility of collective symmetry restoration interacts well with the ideas in the field of relational ontology. Relational ontology highlights an important ontological role of relations. The ontological primacy is not given to individual entities, as in traditional metaphysics, but to relational structures and transformative relational processes (interactions). When accepting this assumption, we cannot imagine the situation when the actor's mind could act absolutely independently and leave all of its relations as assumed in the compatibilist theory of free will. We argue that creative free action can be performed even in the case when the actor is entangled within their material, environmental, and social relational structures.
\end{abstract}

Keywords: creativity, quantum, relational ontology, philosophy of mind, free will

\section{INTRODUCTION}

the philosophy of mind faces the big challenge of how to explain the instance when something new is coming into being in the world. It has much to do with the issue of creative acts. A creative act itself denotes a creative momentum when some novelty, or even something fundamentally new occurs. This moment is also called the "Aha!" experience or the "eureka" moment when taking the creative outputs of reasoning into consideration. However, one may ask as to what possible mechanism is responsible for 
the creative act. This question is a question targeting the process of generating novel, original ideas or experiences. A better understanding of the creative act is exceptionally important, given the fact that most of essential human discoveries, as well as exceptional artistic artefacts, have been accompanied by the creativity of human mind.

The very recent progress in the philosophy of mind focused on creativity is closely related to the theoretical background of theoretical physics, more specifically, to the concept of spontaneous symmetry breaking within quantum field theory [1]. These insights are very urgent, and the present study aims to discuss the important implications that the concept of spontaneous symmetry breaking has for the philosophy of mind and other fields. Firstly, the concept of spontaneous symmetry breaking will be briefly introduced to provide readers basic orientation in this field. The application of temporary symmetry restoration [1] for explaining the essence of the creative act will be also presented. We expand on the argument of symmetry restoration with the interaction of mind within the realm of all possibilities, i.e. within the realm of potentiality, as discussed in our previous work [2]. Furthermore, implications for several fields of philosophy will be drawn from the application of symmetry restoration in modelling the creative act, i.e. for relational ontology, for the theory of free will, and virtue epistemology. Finally, we conclude the present study with concluding remarks considering the dynamics of the realm of potentiality.

\section{State of mind, symmetry restoration, and realm of potentiality}

Recent advances in the philosophy of mind indicate that an in-depth understanding of the creative act can be reached with the help of quantum theory. More specifically, there are insights indicating that creative momentum has many similarities with the concept of spontaneous symmetry breaking, or more specifically, with temporary symmetry restoration [1]. In theoretical physics (in quantum field theory), symmetry refers to invariance in transformation [1], [3]. At the beginning, it is necessary to point out that perpetual transformations occur in minds, as well as in other systems during the flow of time [2]. Minds perpetually generate new thoughts, and they are also perpetually changing. They are in a constantly changing flux [2], i.e. in the process of flowing movement [4].

When cognition remains in the state of asymmetry, restrictions for admissible transformations are present [1]. Such restrictions are, for example, the stable knowledge categories, rigid expectations about objects and entities in the world, subjective concepts based on previous experience, or rigid social scripts. These restrictions maintain the mind in the state of asymmetry, and block any transformations that may possibly lead to emergence of creative thought.

The human mind needs a sort of order in the chaotic and perpetually changing world. Therefore, stable knowledge categories about objects and their behavior develop in the mind as children grow up. These internalized mental schemes help people orient themselves easily in the world and to act effectively. They become more rigid when elderly, with their increasing experience with the world. Mental schemes that have been proven to be valid through personal experience and practice are considered to be functional and thus need not be changed. Thus, with increasing age, restrictions for admissible transformations become more stable and are less likely to be overcome.

During the flowing time flux, there are moments when the mind secedes from its ordinary way of thinking, and produces novel or even fundamentally new thoughts. 
These kinds of moments are called, for example, the "Aha!" experience or the "eureka" moment when analyzing the creative products of reasoning. In terms of quantum theory, this moment is the moment that may be called temporary symmetry restoration [1]. Temporary symmetry restoration means that one's cognition may leave, at least temporarily, conventional thinking and stable categories, heuristics, etc. It means that one's cognition shifts, although for just a limited time period, to the state of symmetry. In this moment, a novel, original idea may arise. Creative momentum can be compared to a kind of sudden enlightenment or spontaneous emergence.

The state of symmetry is generally characterized by a superposition of possibilities [1]. In the symmetric state, all instances are possible. Symmetry admits all possible instances, even such instances that go against our common, everyday experience, e.g. the instance when a cat can be considered to be both dead and alive at the same moment. The symmetric state thus enables cognition to accept all possible instances.

Here, we expand upon the argument of symmetry restoration, which is about understanding of the mental creative event as a moment when the mind gets into contact with the realm of all possibilities, i.e. with the realm of potentiality in terms of quantum anthropology [2]. Following the Aristotelian differentiation between actus and potentia, one can distinguish two different realms. The realm of potentiality refers to the hidden, invisible domain of all possibilities. It is the source of all transformations, emergences, patterns, ideas, etc. In contrast, the realm of actuality is the domain of our reality, including systems, entities, and ideas that have been already actualized in time and space. When framing temporary symmetry restoration in cognition within the framework of quantum anthropology [2], symmetry restoration can be understood as a process accompanying the interaction between potentiality and actuality. When a new thought comes into being, it arises from the overall number of all possibilities and emerges in one's mind. We argue that during creative momentum, the mind touches the realm of potentiality. Hence, the creative act is suggested to be a particular actualization in time and space.

\section{Strong creativity and emergence}

The connection of creative momentum with the realm of potentiality [2] is related to the notion of creative dispositional potentialities. Abel [5] speaks about creative dispositional potentialities when exploring the nature of strong creativity, i.e. creativity that refers to the emergence of fundamentally new ideas, products, and entities. Strong (or radical) creativity:

a) should not be reduced to a set of antecedent elements and their principles and rules

(which are violated and superseded in radical creativity)

b) cannot be deduced logically, causally, or psychologically from such a given set of elements

c) is suggested to be linked more to "luck" (in the sense of randomness) than to reason

d) is suggested to be spontaneous

e) is suggested to be unpredictable, and

f) is suggested to be characterized by discontinuities [5]

All of the aforementioned features indicate that the creative act has the hallmarks of emergence: 
"Emergence is a generation process whereby a produced phenomenon (the 'emergent') is not completely reducible to the set of previous phenomena from which it was generated (the 'emergence base'), in the sense that it represents a qualitative novelty and manifests some degree of reality or autonomy over and above the set of its base elements. That is, although an emergent phenomenon comes into existence as a result of some set of previous elements in relation, an emergent is never a mere derivative epiphenomenon of a production process, having its own ontological status, identity and causal efficacy." [p. 430, 6]

As mentioned above, Sundararajan and Fatemi [1] explain the emergence of new creative ideas by the process of symmetry restoration. Symmetry restoration occurs when a system in an asymmetric state shifts into a symmetric state. For example, conventional thinking may be considered to be processes occurring in the mind in order to maintain asymmetry [1]. In other words, conventional thinking can be metaphorically compared to a kind of "frozen asymmetry". The mind works with stable knowledge categories, stable expectations about objects and entities in the world, and subjective concepts based on previous experience. In the moment when conventional order and structures in cognition are undermined, spontaneous symmetry restoration occurs in cognition [1]. Thus, spontaneous symmetry breakdown denotes a kind of process that enables the emergence of something fundamentally new, something that has never been thought or done before.

\section{IMPLICATIONS FOR THE PHILOSOPHY OF MIND}

\section{The creative act, insightfulness, and virtue epistemology}

From the perspective of virtue epistemology, the emergence of deep, non-trivial, original thought is related to a kind of insight [7]. The episode of insight is a mental event in which one achieves insight in the object sense. Insight is connected to some epistemic end (i.e. truth), and it is suggested to be often linked to the discovery of solutions. During an episode of insight, we suddenly see a solution that was previously not available to our consciousness. It is a kind of "Aha!" experience or "eureka" moment. Interestingly, these events are suggested to be linked to illumination and to experiencing clarity.

Carter [7] considers insightfulness to be a paradigmatic intellectual virtue. Insightfulness is suggested to be a trait disposition for achieving insightful episodes relating to creation of deep, non-trivial, original thought. People are suggested to be more or less insightful, depending on the frequency, depth, non-triviality, and originality of emerging thoughts.

If we admit that the episode of insight, i.e. the emergence of non-trivial, original thought, is not gradual but sudden [7], one may ask about the role of cognition in the emergence of insight. Carter points out that people often do not think about the possible solutions of a problem at the moment when the solution spontaneously emerges. The emergence of insight is suggested to be sudden and non-conscious [7]. Similarly, the emergence of fundamentally new ideas is connected with a spontaneous, unpredictable, and discontinuous mental event [5]. Fundamentally new ideas are not deduced logically or causally from previously known antecedent elements and given rules and categories. They emerge spontaneously and are often linked more to random coincidence rather 
than to reason. All of these arguments indicate that such types of emergence may be conceptualized as spontaneous temporary symmetry restoration. In contrast, the insight that is an outcome of previous conscious cognitive processing has a different character.

We argue that the emergence of insight that is the result of conscious cognitive processing does not have the character of strong, radical creativity [5]. In strong creativity, the output of a creative mental event should not be deduced from a given set of antecedent elements existing previously in mind. We should distinguish two instances, a) the emergence of genuinely new, original thought and b) the emergence of novel thought. Genuinely new, original thought is linked to the agency of strong, radical creativity, whereas the emergence of novel thought is still the result of a set of antecedent elements existing previously in mind, e.g. conventional conceptual categories and expectations. Consequently, within the framework of virtue epistemology, we argue that there are more types of insights. Some insights are related to the emergence of genuinely new, original thoughts, whereas other insights are merely novel thoughts resulting from the unique combination of antecedent elements. Despite of this difference, both instances share similar characteristics in terms of spontaneous occurrence and unpredictability, and they may be, therefore, connected with spontaneous temporary symmetry restoration.

To differentiate between the emergence of new, original thought and the emergence of novel thought is very important, because it also elicits the question concerning the difference related to the motivation for the creative engaging of one's mind. We argue that we should differentiate between "insight for the sake of problem solving" and "thinking without a goal". Emergent phenomena are characterized by an absence of either the starting or the ending situation [5]. However, if we consider an insight that is linked to the discovery of a solution, it means that such an insight is not end-free. In contrast, the emergence of such insight was motivated by the conscious (or unconscious) effort to solve the particular problem. Therefore, such a result of thinking may be deduced logically or causally, and it also has a continuity with previous reasoning. As such, "insight for the sake of problem solving" indeed does not have all characteristics of emergent phenomena. Such insight may occur suddenly, spontaneously, and without actual motivation to solve the problem, but we cannot speak of an absence of ending situation or continuity. In contrast, the emergence of fundamentally new, original insight is suggested to be rather the result of "thinking without a goal". In this case, it is justified to speak about insight that has the character of emergent phenomena.

\section{Free will, independence, and creative free action}

The compatibilist theory of free will generally claims that agents may act freely under a specific set of initial conditions such as authenticity or independence [8]. The moment of the emergence of original, creative thought is linked with the requirement of independence. The mind of an agent may be engaged in creative free action only if it reaches a state of independence [8]. The independence of mind denotes the absence of any causal influences of previous thoughts or products [8], [9]. Only if such state of independence is reached can genuine originality emerge in an agent's thoughts and products.

The requirement of independence is in accordance with our previous conceptualizations. Under ordinary conditions, an agent's cognition works with stable knowledge 
categories, stable expectations about objects and entities in the world, and subjective concepts based on previous experience. If an agent is limited by the causal influences of previous thoughts, the emergence of creative free action is not probable. In quantum terms, this state of mind means that an agent's cognition is dependent on the restrictions for admissible transformations and these restrictions block the emergence of creative free action. The emergence of creative free action is more probable under the condition when conventional order and structures in cognition are undermined. At this moment, we can say that cognition moves to a state of independence in terms of the theory of free will. At the same moment, temporary symmetry restoration enables cognition to get in contact with the realm of all possibilities, i.e. with the realm of potentiality in terms of quantum anthropology [2]. Hence, we argue that temporary symmetry restoration in cognition is considered to be an essential prerequisite for the achievement of the state of independence enabling the performance of creative free action.

\section{Relational ontology and the collaborative emergence of creativity}

Barnes [8] targets the initial conditions necessary for the emergence of creative free action mostly on the individual agency of an agent. He points out that creativity requires the ability to think in ways generated largely by one's own mind. Only one's mind is considered to be the primary source of novelty. Equally so, other agents are understood as manipulators who function as a barrier for the emergence of creative free action.

Interestingly, this conceptualization does not admit the instance when creative free action emerges from the interaction between different agents [10]. We may imagine instances of collaborative creative emergence during small-group brainstorming sessions, creative improvisational performances, e.g. improvisational theatre or jazz improvisation. Such emergence of creative free action cannot be reducible to separate agencies of participating agents, but can be rather understood as an outcome of interactions between agents. Aside from the individual agencies of agents, small-group collective agency also plays a role. Trnka and Lorencova [2] understand collective agency as a "supra-individual, collective potential that acts to change reality". Thus, both bottom-up processes and top-down effects are present during the collaborative creative emergence.

Furthermore, man does not live in a vacuum and we cannot imagine the situation when an actor acts absolutely independently, leaving all of their relations. Man lives within relations in their material, environmental, and social anchoring. During the so-called "Aha!" experience or "eureka" moment, one's body, as well as mind, is situated into a particular context and space. Even though one's mind may temporarily leave stable knowledge categories, stable expectations, and subjective concepts, the influences of previous, as well as actual, relations are still present. So many different relations determine man's actions in the world, and the arguments of philosophers in the field of relational ontology [6], [11], [12], [13] are, therefore, eligible.

Relational ontology highlights the important ontological role of relations. According to relational ontology, inspired by the principles of quantum mechanics, ontological primacy is not given to individual entities, as in traditional metaphysics, but to some sorts of relational structures [6], [11]. Transformative relational processes (interactions) between the system's components and between the system and its environment are the foundational basis for any explanation of any form of existence. The same can be applied to the existence-conditions of creative thought and qualitative novelty. Although 
we consider transformative relational interactions in the mind as a primary source of thoughts, interactions between mind and environment should not be neglected. The mind is not closed system. Therefore, existence-conditions for creative thought include qualitatively transformative processes in the mind, as well as interactions between the mind and external environment. Here we see a specific qualitative codependency [6], [11] relating to the initial conditions for the emergence of qualitative novelty. This relatedness of mind is a natural consequence of man's living in a highly complex world. The instance when an actor would be absolutly free of their relations has seemingly been proven to be invalid. Thus, we argue that an absolute independence of mind from relational structures cannot be reached, at least under normal, non-altered states of consciousness.

Consequently, temporary symmetry restoration may occur in the cognition of one agent, but a similar process may also occur during the interaction between the cognitions of two or more agents. In the second case, temporary symmetry restoration occurs during the mutual interaction of two or more minds, and therefore, such instances can be interpreted as moments of collective symmetry restoration. As seen on the examples of small-group brainstorming sessions, improvisational theatre, or jazz improvisation, the co-dependency of relations may have productive agency and generate qualitatively new thoughts or artistic expressions. We conclude that agents are not always barriers for the emergence of creative free action, but that they may be also the active instigators of a collaborative creative momentum.

\section{CONCLUSION}

The present study shows how a creative mental event can be explained in terms of quantum field theory. We argue that temporary symmetry restoration, responsible for emergence of creative thought in mind, is closely related to the moment when one's cognition gets in contact with the realm of potentiality [2]. In contrast to the systemic understanding of emergence, where emergent properties are suggested to be actualizations of qualities already existing at lower levels [6], [10], the quantum anthropological understanding of the realm of potentiality is different [2]. We do not understand the realm of potentiality as a relatively stable set of properties existing at lower levels. The structure of the realm of potentiality is hypothesized to be more dynamic, and rather similar to the idea of quantum sea [14]. Quantum sea is suggested to be a single underlying substructure that is common for the entire universe. This underlying substructure consists of so-called zero-point fluctuations of fields in the vacuum [14]. A vacuum is never particle- or field-free, but consists of continuous virtual particle-pair creation and annihilation. There are fleeting electromagnetic waves and pairs of microparticles that perpetually come into being and return back again into nonexistence [14]. In this perspective, the existence of particular potentialities is only transient. Potentialities are perpetually coming into existence and also vanishing on a dynamic-balance basis. Such conceptualization of the realm of all possibilities does not assume a stability over time nor a relatively stable set of properties existing at lower levels as the basis for emergence. We believe that the contrast between these two conceptualizations of potentialism may stimulate future discussions in this field.

Furthermore, the present study also shows that temporary symmetry restoration may occur not only on the individual level, but also during collaborative creation, when restrictions for admissible transformations are overcome collectively. Considering 
multiple levels of creative emergence would be inspiring for future development in this field.

\section{ACKNOWLEDGMENTS}

Many thanks to Radmila Lorencova for her inspiring comments to the final version of manuscript. This work was supported by the Czech Science Foundation (GACR), project no. 18-26094S "Emotional creativity and cognitive decline in the elderly".

\section{REFERENCES}

[1] Sundararajan, L., \& Fatemi, S. M. (2016). Creativity and symmetry restoration: Toward a cognitive account of mindfulness. Journal of Theoretical and Philosophical Psychology, 36(3), 131-141.

[2] Trnka, R., \& Lorencova, R. (2016). Quantum anthropology: Man, cultures, and groups in a quantum perspective. Prague: Charles University Karolinum Press.

Available at:

https://www.researchgate.net/publication/308792484_Quantum_Anthropology_Man_C ultures_and_Groups_in_a_Quantum_Perspective

[3] Bolender, J. (2010). The self-organizing social mind. Cambridge, MA: MIT Press.

[4] Bohm, D. (1980). Wholeness and implicate order. London: Routledge and Kegan Paul.

[5] Abel, G. (2009). The riddle of creativity: philosophy's view. In: P. Meusburger, J. Funke, \& E. Wunder (Eds.), Milieus of creativity: An interdisciplinary approach to spatiality of creativity (pp. 53-72). Heidelberg: Springer.

[6] Santos, G. C. (2015). Ontological emergence: How is that possible? Towards a new relational ontology. Foundations of Science, 20(4), 429-446.

[7] Carter, J. A. (2017). Virtuous insightfulness. Episteme, 14(4), 539-554.

[8] Barnes, E. C. (2015). Freedom, creativity, and manipulation. Nô̂s, 49(3), 560-588.

[9] Kronsfeldner, M. (2009). Creativity naturalized. The Philosophical Quarterly, 59, 577-592.

[10] Sawyer, R. K. (1999). The emergence of creativity. Philosophical Psychology, 12(4), 447-469.

[11] Cordovil, J. L. (2015). Contemporary quantum physics metaphysical challenge: Looking for a relational metaphysics. Axiomathes, 25(1), 133-143.

[12] Ferrero, M., \& Sánchez-Gómez, J. L. (2015). Coming from material reality. Foundations of Science, 20(2), 199-212.

[13] Paoletti, M. P. (2016). How powers emerge from relations. Axiomathes, 26(2), 187204.

[14] Puthoff, H. E. (2002). Searching for the universal matrix in metaphysics. Research News and Opportunities in Science and Theology, 2(8), 22-24. 